\title{
Biography: Professor Cunchuan Wang, MD, PhD
}

Wah Yang ${ }^{1,2,3,4}$

Published online: 13 February 2019

(C) Springer Science+Business Media, LLC, part of Springer Nature 2019

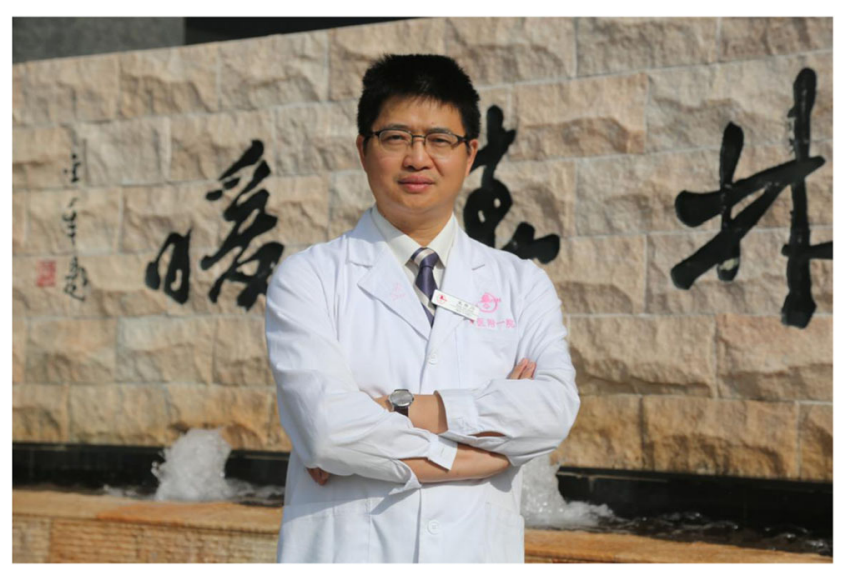

Professor Cunchuan Wang was born in the Sichuan Province in China, the beautiful hometown of the panda. He graduated from Luzhou Medical College (now named as Southwest Medical University) in Sichuan Province, China, and then worked in the Department of General Surgery in Xuanhan People's Hospital for 2 years. After his postgraduate study in Jinan University, Professor Wang started working as a laparoscopic surgeon in the Department of General Surgery, the First Affiliated Hospital of Jinan University in Guangzhou, China.

Wah Yang

yangwah@qq.com

1 Department of Metabolic and Bariatric Surgery, The First Affiliated Hospital, Jinan University, Guangzhou, China

2 Joint Institute of Metabolic Medicine Between State Key Laboratory of Pharmaceutical Biotechnology, The University of Hong Kong and Jinan University, Guangzhou, China

3 State Key Laboratory of Pharmaceutical Biotechnology, LKS Faculty of Medicine, The University of Hong Kong, Hong Kong, China

4 Department of Medicine, LKS Faculty of Medicine, The University of Hong Kong, Hong Kong, China
Later, he obtained his PhD Degree in Huazhong University of Science and Technology Tongji Medical College. Professor Wang is a pioneer of laparoscopic surgery in China. He was also a visiting scholar at Mount Sinai Medical Center in the USA in 2000, where he met Professor Michel Gagner, a world renowned laparoscopic bariatric surgeon.

Professor Wang started performing metabolic and bariatric surgery in China in 2000, becoming the first surgeon to perform the laparoscopic Roux-en-Y gastric bypass (LRYGB) in mainland China. Additionally, Professor Wang performed a LRYGB on a male with a BMI of 99 who was at the time considered as the man with highest BMI in China. Professor Wang also introduced the concept of "Precise metabolic and bariatric surgery" which resulted in better surgical outcomes and safety. In his center, the procedures primarily performed are the LRYGB, laparoscopic sleeve gastrectomy (LSG), reduced ports or single port LRYGB or LSG, and a variety of revisional surgeries, etc. Currently, his center has the largest case volume in China.

Professor Wang initiated the founding of the Chinese Society for Metabolic and Bariatric Surgery (CSMBS) in 2012 with experts from Shenyang and Shanghai. He is currently the President of the CSMBS, Vice-President of the First Affiliated Hospital of Jinan University, and Director of the Department of Metabolic and Bariatric Surgery (International Bariatric Center). As the bariatric expert of China, he also received special government allowances from the State Council in China.

Professor Wang established and is also the Editor-inChief of the first Chinese journal pertaining to the clinical and basic research of metabolic and bariatric surgery named the Chinese Journal of Obesity and Metabolic Diseases. He is also the Editor-in-Chief of the first Chinese textbook on bariatric surgery named Bariatric and Metabolic Surgery. Furthermore, in 2018, Professor Wang included metabolic and bariatric surgery topics into the textbook used by Chinese medical undergraduates. He has also contributed chapters to other books related to laparoscopic surgery, bariatric surgery, and thyroid 
surgery. Professor Wang has published more than 300 articles in Chinese and English peer-reviewed journals. $\mathrm{He}$ has supervised more than 100 master and doctoral postgraduate students and fellows both Chinese nationals and others from around the world.

Professor Wang is a member of the International Federation for the Surgery of Obesity and Metabolic Disorders (IFSO) and an international member of the American Society for Metabolic and Bariatric Surgery (ASMBS). He is also the Executive Board Member of the IFSO-Asia Pacific Chapter (IFSO-APC). He has been invited to deliver presentations or live surgery demonstrations around the world, including the USA, UK, Germany, Japan, France, South Korea, Malaysia, Vietnam, Turkey, Singapore, India, Austria, Egypt, Canada, Sweden, and Brazil, etc.

Professor Wang began performing laparoscopic surgery in 1991, focusing on minimally invasive surgery for obesity and related metabolic diseases, thyroid disease, gastrointestinal carcinoma, hepatobiliary disease, and diseases of pancreas, spleen, and hernia, etc. He has performed more than 100 different laparoscopic procedures in more than 10,000 cases making him one of the highest case volume surgeons in mainland China. These include some advanced procedures such as laparoscopic pancreaticoduodenectomy and laparoscopic right hemihepatectomy. Professor Wang also performed the first cases in mainland China of endoscopic thyroidectomy for hyperthyroidism, complete laparoscopic radical gastrectomy for gastric fundus and cardia cancers, laparoscopic partial hepatectomy for the treatment of intrahepatic cholelithiasis, laparoscopic total colectomy, and laparoscopic Roux-en-Y gastric bypass for obesity and metabolic disorders.

Professor Wang was also the first to perform the endoscopic thyroidectomy via areola of breasts approach
(ETABA) in 2005 and endoscopic thyroidectomy via oral vestibular approach (ETOVA) in 2011. He has performed more than 5000 cases of endoscopic thyroidectomy which is currently the largest number of cases in the world. Professor Wang has made great contributions to the popularization of laparoscopic surgery in China since 1997. $\mathrm{He}$ has given presentations in more than 500 academic conferences. He has been invited to more than 400 hospitals in China to assist them in various minimally invasive operations, such as metabolic and bariatric surgery, general surgery, thoracic surgery, urinary surgery, and gynecology. However, since 2013, he has focused mainly on metabolic and bariatric surgery.

During his 30 years in practice, Professor Wang has devoted himself to patient care, training minimally invasive surgeons and metabolic and bariatric surgeons, and has made outstanding contributions to the development of laparoscopic surgery and metabolic and bariatric surgery in China. Professor Wang is still on the front line of clinical practice and attaches the importance of international academic exchanges in metabolic and bariatric surgery. He has invited many metabolic and bariatric surgeons to come to China for academic exchanges so as to promote the professional training of Chinese surgeons and to lead the development of metabolic and bariatric surgery in China to the world.

Professor Wang and his wife, Dr. Li Wang, met in high school and have a daughter who is currently working as an accountant in Australia. Professor Wang enjoyed Chinese Kung Fu when he was young, and he has a deep understanding of Chinese history. He also has a strong interest in the history and culture of different countries. When he travels abroad, he enjoys visiting the local historical sites and museums.

Publisher's Note Springer Nature remains neutral with regard to jurisdictional claims in published maps and institutional affiliations. 